\title{
Automatic Stance Detection Using End-to-End Memory Networks
}

\author{
Mitra Mohtarami ${ }^{1}$, Ramy Baly $^{1}$, James Glass $^{1}$, Preslav Nakov $^{2}$ \\ Lluís Màrquez ${ }^{3 *}$,Alessandro Moschitti ${ }^{3 *}$ \\ ${ }^{1}$ MIT Computer Science and Artificial Intelligence Laboratory, Cambridge, MA, USA \\ ${ }^{2}$ Qatar Computing Research Institute, HBKU, Doha, Qatar; ${ }^{3}$ Amazon \\ \{mitra,baly, glass\}@csail.mit.edu \\ pnakov@hbku.edu.qa; \{lluismv, amosch\}@amazon.com
}

\begin{abstract}
We present an effective end-to-end memory network model that jointly $(i)$ predicts whether a given document can be considered as relevant evidence for a given claim, and (ii) extracts snippets of evidence that can be used to reason about the factuality of the target claim. Our model combines the advantages of convolutional and recurrent neural networks as part of a memory network. We further introduce a similarity matrix at the inference level of the memory network in order to extract snippets of evidence for input claims more accurately. Our experiments on a public benchmark dataset, FakeNewsChallenge, demonstrate the effectiveness of our approach.
\end{abstract}

\section{Introduction}

Recently, an unprecedented amount of false information has been flooding the Internet with aims ranging from affecting individual people's beliefs and decisions (Mihaylov et al., 2015; Mihaylov and Nakov, 2016) to influencing major events such as political elections (Vosoughi et al., 2018). Consequently, manual fact checking has emerged with the promise to support accurate and unbiased analysis of rumors spreading in social medias, as well as of claims made by public figures or news media.

As manual fact checking is a very tedious task, automatic fact checking has been proposed as a possible alternative. This is often broken into intermediate steps in order to alleviate the task complexity. One such step is stance detection, which is also useful for human experts as a stand-alone task. The task aims to identify the relative perspective of a piece of text with respect to a claim, typically modeled using labels such as agree, disagree, discuss, and unrelated; Figure 1 gives some examples.

\footnotetext{
${ }^{*}$ Work conducted while these authors were at QCRI
}

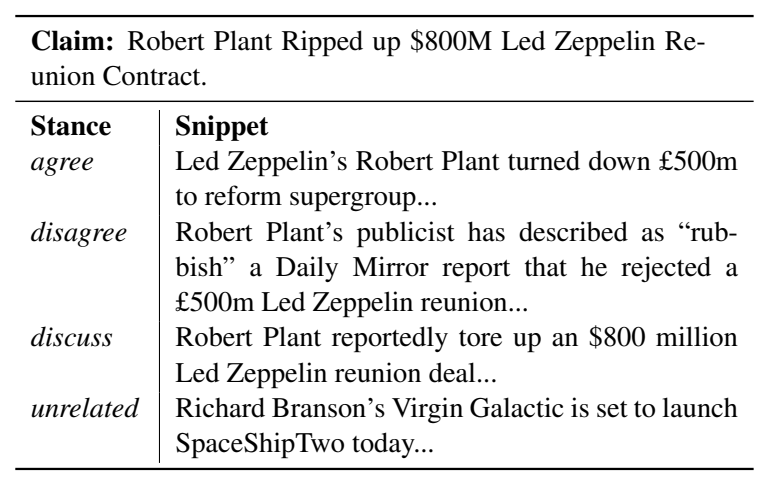

Figure 1: Examples of snippets of text and their stance with respect to the target claim.

Here, we address the problem of stance detection using a novel model based on end-to-end memory networks (Sukhbaatar et al., 2015), which incorporates convolutional and recurrent neural networks, as well as a similarity matrix. Our model jointly addresses the problems of predicting the stance of a text with respect to a given claim, and of extracting relevant text snippets as support for the prediction of the model. We further introduce a similarity matrix, which we use at inference time in order to improve the extraction of relevant snippets.

The experimental results on the Fake News Challenge benchmark dataset show that our model, which is very feature-light, performs close to the state of the art. Our contributions can be summarized as follows: (i) We apply a novel memory network model enhanced with $\mathrm{CNN}$ and LSTM networks for stance detection. (ii) We further propose a novel extension of the general architecture based on a similarity matrix, which we use at inference time, and we show that this extension offers sizable performance gains. (iii) Finally, we show that our model is capable of extracting meaningful snippets from a given text document, which is useful not only for stance detection, but more importantly can support human experts who need to decide on the factuality of a given claim. 


\section{Stance Detection Memory Networks}

Long-term memory is necessary to determine the stance of a long document with respect to a claim, as relevant parts of a document-paragraphs or text snippets - can indicate the perspective of a document with respect to a claim. Memory networks have been designed to remember past information (Sukhbaatar et al., 2015) and they can be particularly well-suited for stance detection since they can use a variety of inference strategies alongside their memory component.

In this section, we present a novel memory network for stance detection. It contains a new inference component that incorporates a similarity matrix to extract, with better accuracy, textual snippets that are relevant to the input claims.

\subsection{Overview of the network}

A memory network is a 5-tuple $\{M, I, G, O, R\}$, where the memory $M$ is a sequence of objects or representations, the input $I$ is a component that maps the input to its representation, the generalization component $G$ (Sukhbaatar et al., 2015) updates the memory with respect to new input, the output $O$ generates an output for each new input and the current memory state, and finally, the response $R$ converts the output into a desired response format, e.g., a textual response or an action. These components can potentially use many different machine learning models.

Our new memory network for stance detection is a 6-tuple $\{M, I, F, G, O, R\}$, where $F$ represents the new inference component. It takes an input document $d$ as evidence and a textual statement $s$ as a claim and converts them into their corresponding representations in the input $I$. Then, it passes them to the memory $M$. Next, the relevant parts of the input are identified in $F$, and afterwards they are used by $G$ to update the memory. Finally, $O$ generates an output from the updated memory, and converts it to a desired response format with $R$. The network architecture is depicted in Figure 2. We describe the components below.

\subsection{Input Representation Component}

The input to the stance detection algorithm is a document $d$ as evidence and a textual statement $s$ as a claim, (see lines 2 and 3 in Table 1). Each $d$ is segmented into paragraphs $x_{j}$ of varied lengths, where each $x_{j}$ is considered as a piece of evidence for stance detection.

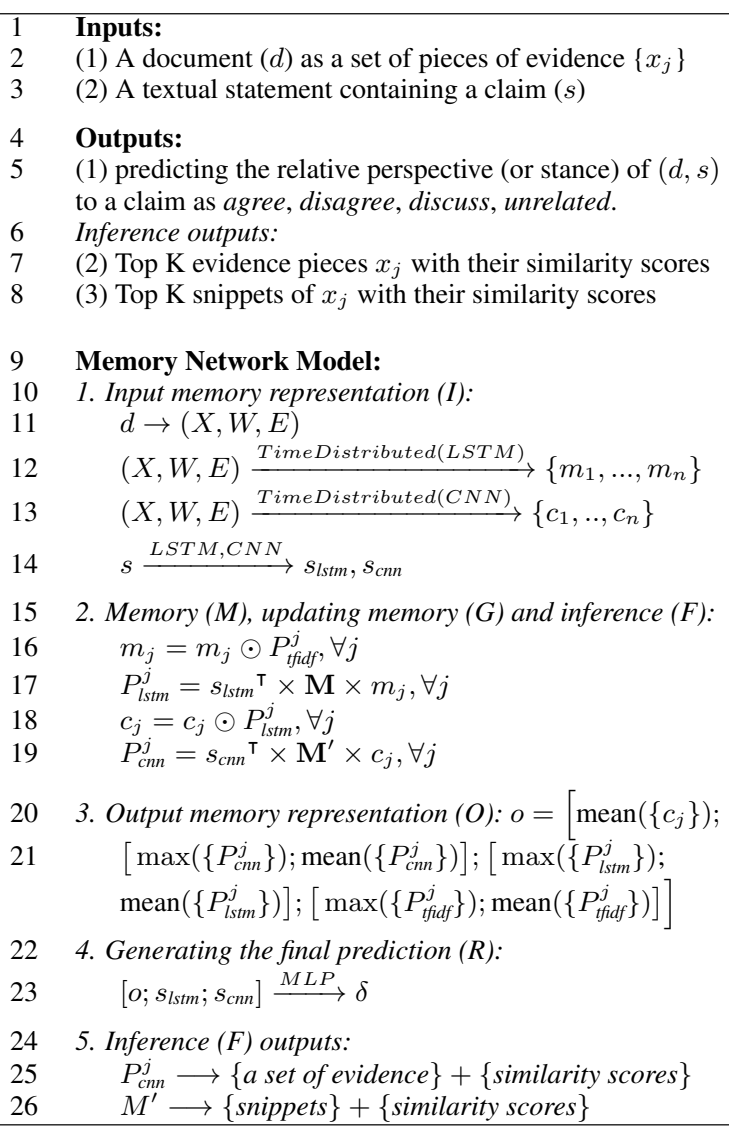

(1) A document $(d)$ as a set of pieces of evidence $\left\{x_{j}\right\}$

(2) A textual statement containing a claim $(s)$

Outputs:

(1) predicting the relative perspective (or stance) of $(d, s)$ to a claim as agree, disagree, discuss, unrelated. Inference outputs:

(2) Top K evidence pieces $x_{j}$ with their similarity scores

(3) Top K snippets of $x_{j}$ with their similarity scores

\section{Memory Network Model:}

1. Input memory representation (I):

$11 \quad d \rightarrow(X, W, E)$

$12(X, W, E) \stackrel{\text { TimeDistributed }(L S T M)}{\longrightarrow}\left\{m_{1}, \ldots, m_{n}\right\}$

$13(X, W, E) \stackrel{\text { TimeDistributed }(C N N)}{\longrightarrow}\left\{c_{1}, . ., c_{n}\right\}$

$14 \stackrel{\operatorname{LSTM,CNN} \longrightarrow}{\longrightarrow} s_{l s t m}, s_{c n n}$

15 2. Memory $(M)$, updating memory $(G)$ and inference $(F)$ : $m_{j}=m_{j} \odot P_{t f i d f}^{j}, \forall j$

$P_{l s t m}^{j}=s_{l s t m}{ }^{\top} \times \mathbf{M} \times m_{j}, \forall j$

$c_{j}=c_{j} \odot P_{l s t m}^{j}, \forall j$

$P_{c n n}^{j}=s_{c n n}^{\top} \times \mathbf{M}^{\prime} \times c_{j}, \forall j$

3. Output memory representation $(O): o=\left[\operatorname{mean}\left(\left\{c_{j}\right\}\right)\right.$; $\left[\max \left(\left\{P_{c n n}^{j}\right\}\right) ; \operatorname{mean}\left(\left\{P_{c n n}^{j}\right\}\right)\right] ;\left[\max \left(\left\{P_{l s t m}^{j}\right\}\right) ;\right.$ $\left.\left.\operatorname{mean}\left(\left\{P_{\text {lstm }}^{j}\right\}\right)\right] ;\left[\max \left(\left\{P_{t \text { tidf }}^{j}\right\}\right) ; \operatorname{mean}\left(\left\{P_{\text {tfidf }}^{j}\right\}\right)\right]\right]$

22 4. Generating the final prediction $(R)$ : $\left[o ; s_{l s t m} ; s_{c n n}\right] \stackrel{M L P}{\longrightarrow} \delta$

5. Inference $(F)$ outputs:

$P_{c n n}^{j} \longrightarrow\{$ a set of evidence $\}+\{$ similarity scores $\}$ $M^{\prime} \longrightarrow\{$ snippets $\}+\{$ similarity scores $\}$

Table 1: Summary of our memory network algorithm for stance detection.

Indeed, a paragraph usually presents a coherent argument, unified under one or more inter-related topics. The input component in our model converts each $d$ into a set of pieces of evidence in a three dimensional (3D) tensor space as shown below (see line 11 in Table 1):

$$
d=(X, W, E)
$$

where $X=\left\{x_{1}, \ldots, x_{n}\right\}$ is a set of paragraphs considered as pieces of evidence; each $x_{j}$ is represented by a set of words $W=\left\{w_{1}, \ldots, w_{v}\right\}-$ drawn from a global vocabulary of size $v$-and a set of neural representations $E=\left\{e_{1}, \ldots, e_{v}\right\}$ for words in $W$. This $3 \mathrm{D}$ space is illustrated as a cube in Figure 2.

Each $x_{j}$ is encoded from the 3D space into a semantic representation at the input component using a Long Short-Term Memory (LSTM) network. The lower left component in Figure 2 shows our LSTM network, which operates on our input as follows (see also line 12 in Table 1):

$$
(X, W, E) \stackrel{\text { TimeDistributed }(L S T M)}{\longrightarrow}\left\{m_{1}, \ldots, m_{n}\right\}
$$




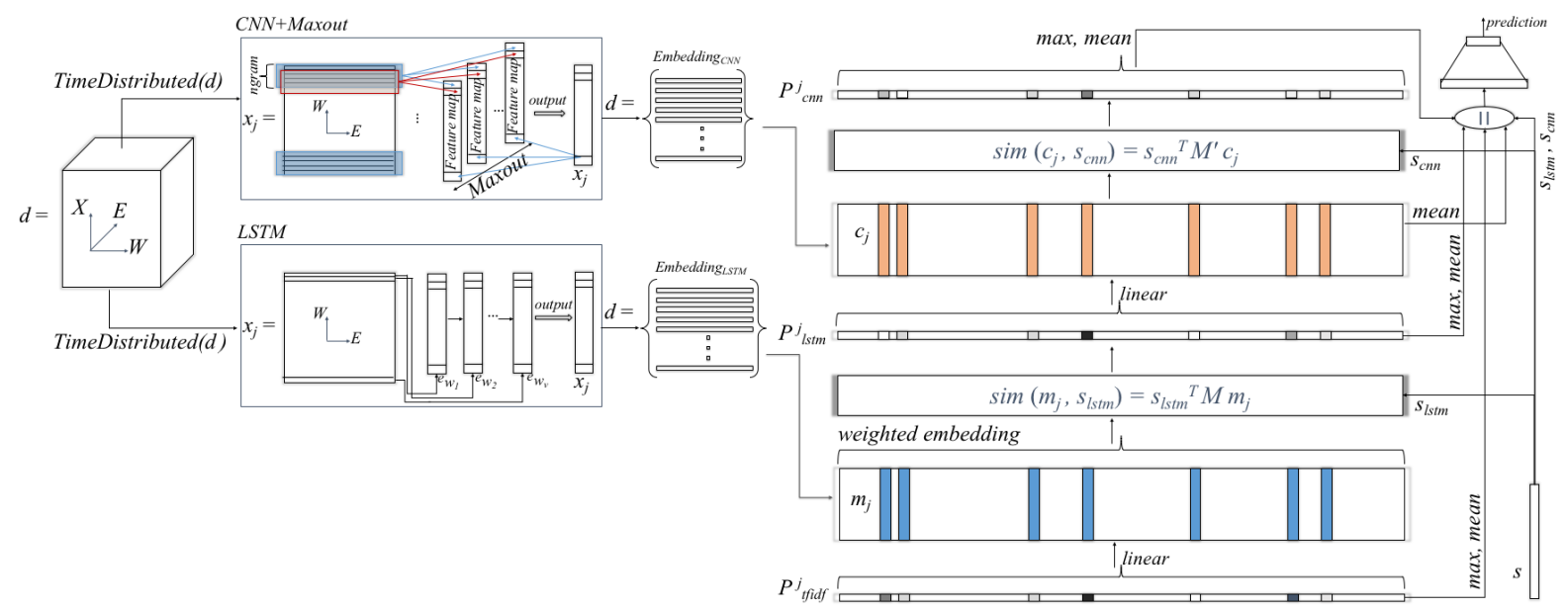

Figure 2: The architecture of our memory network model for stance detection.

where $m_{j}$ is the LSTM representation of $x_{j}$, and TimeDistributed () indicates a wrapper that enables training the LSTM over all pieces of evidence by applying the same LSTM model to each time-step of a 3D input tensor, i.e., $(X, W, E)$.

While LSTM networks were designed to effectively capture and memorize their inputs (Tan et al., 2016), Convolutional Neural Networks (CNNs) emphasize the local interaction between the individual words in the input word sequence, which is important for obtaining an effective representation. Here, we use a $\mathrm{CNN}$ in order to encode each $x_{j}$ into its representation $c_{j}$ as shown below (see line 13 in Table 1).

$$
(X, W, E) \stackrel{\text { TimeDistributed }(C N N)}{\longrightarrow}\left\{c_{1}, . ., c_{n}\right\}
$$

As shown in the left-top corner of Figure 2, this representation is passed as a new input to the component $M$ of our memory network model. Moreover, we keep track of the computed $n$-grams from the CNN so that we can use them later in the inference and in the response components (see sections 2.3 and 2.6). For this purpose, we use a Maxout layer (Goodfellow et al., 2013) to take the maximum across $k$ affine feature maps computed by the $\mathrm{CNN}$, i.e., pooling across channels.

Previous work investigated the combination of convolutional and recurrent representations, which were fed to the other network as input (Tan et al., 2016; Donahue et al., 2015; Zuo et al., 2015; Sainath et al., 2015). In contrast, we feed individual outputs into our memory network separately, and we let it decide which representation better helps the target task. We demonstrate the effectiveness of this choice in our experiments.
Furthermore, we convert each input claim $s$ into its representation using the corresponding LSTM and CNN networks as follows:

$$
s \stackrel{L S T M, C N N}{\longrightarrow} s_{l s t m}, s_{c n n}
$$

where $s_{l s t m}$ and $s_{c n n}$ are the representations of $s$ computed using $L S T M$ and $C N N$ networks, respectively. Note that these are separate networks with different parameters from those used to encode the pieces of evidence.

Lines 10-14 of Table 1 describe the above steps in representing $I$ in our memory network. This component encodes each input document $d$ into a set of pieces of evidence $\left\{x_{j}\right\} \forall j$ : it computes LSTM and CNN representations, $m_{j}$ and $c_{j}$, respectively, for each $x_{j}$, and LSTM and CNN representations, $s_{l s t m}$ and $s_{c n n}$, for each claim $s$.

\subsection{Inference Component}

The resulting representations can serve to compute semantic similarity between claims and pieces of evidence. We define the similarity $P_{l s t m}^{j}$ between $s$ and $x_{j}$ as follows (see line 17 in Table 1):

$$
P_{l s t m}^{j}=s_{l s t m}^{\top} \times \mathbf{M} \times m_{j}, \forall j
$$

where $s_{\text {lstm }} \in \mathbb{R}^{q}$ and $m_{j} \in \mathbb{R}^{d}$ are the LSTM representations of $s$ and $x_{j}$, respectively, and $M \in$ $\mathbb{R}^{q \times d}$ is a similarity matrix capturing their similarity. For this purpose, $M$ maps $s$ and $x_{j}$ into the same space as shown in Figure 3. $M$ is a set of $q \times d$ parameters of the network, which are optimized during the training.

In a similar fashion, we compute the similarity $P_{c n n}^{j}$ between $x_{j}$ and $s$ using the CNN representations as follows (see line 19 in Table 1):

$$
P_{c n n}^{j}=s_{c n n}^{\top} \times \mathbf{M}^{\prime} \times c_{j}, \forall j
$$




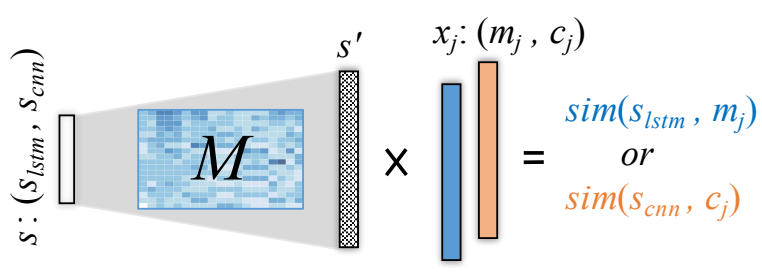

Figure 3: Matching a claim $s$ and a piece of evidence $x_{j}$ using a similarity matrix $M$. Here, $s_{l s t m}$ and $s_{c n n}$ are the LSTM and CNN representations of $s$, whereas $m_{j}$ and $c_{j}$ are the LSTM and CNN representations of $x_{j}$.

where $s_{c n n} \in \mathbb{R}^{q^{\prime}}$ and $c_{j} \in \mathbb{R}^{d^{\prime}}$ are the representations of $s$ and $x_{j}$ obtained with CNN, respectively. The similarity matrix $M^{\prime} \in \mathbb{R}^{q^{\prime} \times d^{\prime}}$ is a set of $q^{\prime} \times d^{\prime}$ parameters of the network and is optimized during the training. $P_{l s t m}^{j}$ and $P_{c n n}^{j}$ indicate the claim-evidence similarity vectors computed based on the LSTM and on the CNN representations of $s$ and $x_{j}$, respectively.

The rationale behind using the similarity matrix is that in our memory network model, as Figure 3 shows, we seek a transformation of the input claim such that $s^{\prime}=M \times s$ in order to obtain the closest facts to the claim.

In fact, the relevant parts of the input document with respect to the input claim can be captured at a different level, e.g., using $M^{\prime}$ for the $n$-gram level or using the claim-evidence $P_{l s t m}^{j}$ or $P_{c n n}^{j}, \forall j$ at the paragraph level. We note that $(i) P_{l s t m}^{j}$ uses LSTM to take the word order and long-length dependencies into account, and (ii) $P_{c n n}^{j}$ uses CNN to take $n$-grams and local dependencies into account, as explained in sections 2.2 and 2.3. Additionally, we compute another semantic similarity vector, $P_{t f i d f}^{j}$, by applying a cosine similarity between the TF.IDF (Spärck Jones, 2004) representation of $x_{j}$ and $s$. This is particularly useful for stance detection as it can help detect unrelated pieces of evidence.

\subsection{Memory and Generalization Components}

The information flow and updates in the memory is as follows: first, the representation vector $\left\{m_{j}\right\} \forall j$ is passed to the memory and updated using the claim-evidence similarity vector $\left\{P_{t f i d f}^{j}\right\}$ :

$$
m_{j}=m_{j} \odot P_{t f i d f}^{j}, \forall j
$$

The reason for this weighting is to filter out most unrelated evidence with respect to the claim. The updated $m_{j}$ in conjunction with $s_{l s t m}$ are used by the inference component-component $F$ to compute $\left\{P_{\text {lstm }}^{j}\right\}$ as explained in Section 2.3.
Then, $\left\{P_{l s t m}^{j}\right\}$ is used to update the new input set $\left\{c_{j}\right\} \forall j$ to the memory:

$$
c_{j}=c_{j} \odot P_{l s t m}^{j}, \forall j
$$

Finally, the updated $c_{j}$ in conjunction with $s_{c n n}$ are used to compute $P_{c n n}^{j}$ as explained in Sec. 2.3.

\subsection{Output Representation Component}

In memory networks, the memory output depends on the final goal, which, in our case, is to detect the relative perspective of a document to a claim. For this purpose, we apply the following equation:

$$
\begin{aligned}
& o=\left[\operatorname{mean}\left(\left\{c_{j}\right\}\right) ;\right. \\
& {\left[\max \left(\left\{P_{c n n}^{j}\right\}\right) ; \operatorname{mean}\left(\left\{P_{c n n}^{j}\right\}\right)\right] ;\left[\max \left(\left\{P_{l s t m}^{j}\right\}\right) ;\right.} \\
& \left.\left.\operatorname{mean}\left(\left\{P_{l s t m}^{j}\right\}\right)\right] ;\left[\max \left(\left\{P_{t f i d f}^{j}\right\}\right) ; \operatorname{mean}\left(\left\{P_{\text {tfidf }}^{j}\right\}\right)\right]\right]
\end{aligned}
$$

where mean $\left(\left\{c_{j}\right\}\right)$ is the average vector of $c_{j}$ representations. Furthermore, we compute the maximum and the average similarity between each piece of evidence and the claim using $P_{t f i d f}^{j}, P_{l s t m}^{j}$ and $P_{c n n}^{j}$, which are computed for each evidence and claim in the inference component $F$. The maximum similarity identifies the part of document $x_{j}$ that is most similar to the claim, while the average similarity measures the overall similarity between the document and the claim.

\subsection{Response and Output Generation}

This component computes the final stance of a document with respect to a claim. For this purpose, the concatenation of vectors $o, s_{l s t m}$ and $s_{c n n}$ is fed into a Multi-Layer Perceptron (MLP), where a softmax predicts the stance of the document with respect to the claim, as shown below (see also lines 22-23 in Table 1):

$$
\left[o ; s_{l s t m} ; s_{c n n}\right] \stackrel{M L P}{\longrightarrow} \delta
$$

where $\delta$ is a softmax function. In addition to the resulting stance, we extract snippets from the input document that best indicate the perspective of the document with respect to the claim. For this purpose, we use $P_{c n n}^{j}$ and $M^{\prime}$ as explained in Section 2.3 (see also lines 24-26 in Table 1).

The overall model is shown in Figure 2 and a summary of the model is presented in Table 1. All the model parameters, including those of $(i) \mathrm{CNN}$ and LSTM in $I$, (ii) the similarity matrices $M$ and $M^{\prime}$ in $F$, and (iii) the MLP in $R$, are jointly learned during the training process. 


\section{Experiments and Evaluation}

\subsection{Data}

We use the dataset provided by the Fake News Challenge, ${ }^{1}$ where each example consists of a claim-document pair with the following possible relations between them: agree (the document agrees with the claim), disagree (the document disagrees with the claim), discuss (the document discusses the same topic as the claim, but does not take a stance with respect to the claim), unrelated (the document discusses a different topic than the topic of the claim). The data includes a total of $75.4 \mathrm{~K}$ claim-document pairs, which link $2.5 \mathrm{~K}$ unique articles with $2.5 \mathrm{~K}$ unique claims, i.e., each claim is associated with 29.8 articles on average.

\subsection{Settings}

We use 100-dimensional word embeddings from GloVe (Pennington et al., 2014), which were trained on two billion tweets. We further use Adam as an optimizer and categorical crossentropy as a loss. We use 100-dimensional units for the LSTM embeddings, and 100 feature maps with filter width of 5 for the CNN. We consider the first $p=9$ paragraphs for each document, where $p$ is the median of the number of paragraphs.

We optimize the hyper-parameters of the models using a validation dataset (20\% of the training data). Finally, as the data is largely imbalanced towards the unrelated class, during training, we randomly select an equal number of instances from each class at each epoch.

\subsection{Evaluation Measures}

We use the following evaluation measures:

Accuracy: The number of correctly classified examples divided by the total number of examples. It is equivalent to micro-averaged $\mathrm{F}_{1}$.

Macro-F1: We calculate $F_{1}$ for each class, and then we average across all classes.

Weighted Accuracy: This is a weighted, twolevel scoring scheme, which is applied to each test example. First, if the example is from the unrelated class and the model correctly predicts it, the score is incremented by 0.25 ; otherwise, if the example is related and the model predicts agree, disagree, or discuss, the score is incremented by 0.25 . Second, there is a further increment by 0.75 for each related example if the model predicts the correct label: agree, disagree, or discuss.

\footnotetext{
${ }^{1}$ Available at www. fakenewschal lenge. org
}

Finally, the score is normalized by dividing it by the total number of test examples. The rationale behind this metric is that the binary related/unrelated classification task is expected to be much easier, while also being arguably less relevant to fake news detection than the stance detection task, which aims to further classify relevant instances as agree, disagree, or discuss. Therefore, the former task is given less weight and the latter task is given more weight through the weighted accuracy metric.

\subsection{Baselines}

Given the imbalanced nature of our data, we use two baselines in which we label all testing examples with the same label: (i) unrelated and (ii) discuss. The former is the majority class baseline, which is a reasonable baseline for Accuracy and macro- $F_{1}$, while the latter is a potentially better baseline for Weighted Accuracy.

We further use CNN and LSTM, and combinations thereof as baselines, since they form components of our model, and also because they yield state-of-the-art results for text, image, and video classification (Tan et al., 2016; Donahue et al., 2015; Zuo et al., 2015; Sainath et al., 2015).

Finally, we include the official baseline from the challenge, which is a Gradient Boosting classifier with word and $n$-gram overlap features, as well as indicators for refutation and polarity.

\subsection{Our Models}

sMemNN: This is our model presented in Figure 2. Note that unlike the CNN+LSTM and the LSTM+CNN baselines above, which feed the output of one network into the other one, the sMemNN model feeds the individual outputs of both the CNN and the LSTM networks into the memory network, and lets it decide how much to rely on each of them. This consideration also facilitates reasoning and explaining model predictions, as we will discuss in more detail below.

sMemNN (dotProduct): This is a version of sMemNN, where the similarity matrices are replaced by the dot product between the representation of the claims and of the evidence. For this purpose, we first project the claim representation to a dense layer that has the same size as the representation of each piece of evidence, and then we compute the dot product between the resulting representation and the representation of the evidence. 


\begin{tabular}{lcc|ccc}
\hline Methods & $\begin{array}{l}\text { Total } \\
\text { Parameters }\end{array}$ & $\begin{array}{l}\text { Trainable } \\
\text { Parameters }\end{array}$ & $\begin{array}{c}\text { Weighted } \\
\text { Accuracy }\end{array}$ & Macro-F1 & Accuracy \\
\hline 1. All-unrelated & - & - & 39.37 & 20.96 & 72.20 \\
2. All-discuss & - & - & 43.89 & 7.47 & 17.57 \\
\hline 3. CNN & $2.7 \mathrm{M}$ & $188.7 \mathrm{~K}$ & 40.66 & 24.44 & 41.53 \\
4. LSTM & $2.8 \mathrm{M}$ & $261.3 \mathrm{~K}$ & 57.23 & 37.23 & 60.21 \\
5. CNN+LSTM & $4.2 \mathrm{M}$ & $361.5 \mathrm{~K}$ & 42.02 & 27.36 & 48.54 \\
6. LSTM+CNN & $2.8 \mathrm{M}$ & $281.5 \mathrm{~K}$ & 60.21 & 40.33 & 65.36 \\
7. Gradient Boosting & - & - & 75.20 & 46.13 & 86.32 \\
\hline 8. sMemNN (dotProduct) & $5.4 \mathrm{M}$ & $275.2 \mathrm{~K}$ & 75.13 & 50.21 & 83.85 \\
9. sMemNN & $5.5 \mathrm{M}$ & $377.5 \mathrm{~K}$ & 78.97 & 56.75 & 87.27 \\
10. sMemNN (with TF) & $110 \mathrm{M}$ & $105 \mathrm{M}$ & $\mathbf{8 1 . 2 3}$ & $\mathbf{5 6 . 8 8}$ & $\mathbf{8 8 . 5 7}$ \\
\hline
\end{tabular}

Table 2: Evaluation results on the test data.

sMemNN (with TF): Since our LSTM and CNN networks use a limited number of starting paragraphs ${ }^{2}$ for an input document, we enrich our model with the BOW representation of documents and claims as well as their TF.IDF-based cosine similarity. We concatenate these vectors with the memory outputs (section 2.5) and pass them to the R component (section 2.6) of sMemNN. We expect these BOW vectors provide useful information that are not initially incorporated into the sMemNN model.

\subsection{Results}

Table 2 reports the performance of all models on the test dataset. The All-unrelated and the Alldiscuss baselines perform poorly across the evaluation measures, except for All-unrelated, which achieves high accuracy, which is due to unrelated being by far the dominant class in the dataset.

Next, we can see that the LSTM model consistently outperforms the CNN across all evaluation measures. Although the larger number of parameters of the LSTM can play a role, we believe that its superiority comes from it being able to remember previously observed relevant pieces of text.

Next, we see systematic improvements for the combinations of the CNN and the LSTM models: CNN+LSTM is better than CNN alone, and LSTM+CNN is better than LSTM alone. Better performance is achieved by the LSTM+CNN model, where claims and evidence are first processed by a LSTM and then fed into a CNN.

The Gradient Boosting model achieves sizable improvement over the above baseline neural models. However, we should note that these neural models do not have the rich hand-crafted features that were used in the Gradient Boosting model.

\footnotetext{
${ }^{2}$ Due to the long length of documents, it is impractical to consider all paragraphs when training LSTM and CNN.
}

Row 9 shows the results for our memory network model (sMemNN), which consistently outperforms all other baseline models across all evaluation metrics, achieving 10.62 and 3.77 points of absolute improvement in terms of Macro-F1 and Weighted Accuracy, respectively, over the best baseline (Gradient Boosting). We believe this is due to the memory network being able to capture good text snippets. As we will see below, these snippets are also useful for explaining the model's predictions. Comparing row 9 to row 8 , we can see the importance of our proposed similarity matrix: replacing that matrix by a simple dot product hurts the performance of the model considerably across all evaluation measures, thus lowering it to the level of the Gradient Boosting model.

Finally, row 10 shows the results for our memory network model enriched by BOW representation. As we expected, it improves the performance of sMemNN - perhaps by capturing useful information from paragraphs beyond the starting few.

To put the results of sMemNN in perspective, we should mention that the best system at the Fake News Challenge (Baird et al., 2017) achieved a macro-F1 of 57.79, which is not significantly different from our results at the 0.05 significance level ( $\mathrm{p}$-value $=0.53$ ). Yet, they have an ensemble combining the feature-rich Gradient Boosting system with neural networks. In contrast, we only use raw text as input and no ensembles, and our main goal is to study a new memory network model and its explainability component.

Further analysis of the outputs (namely, the confusion matrices) of the different models we experimented with reveals the following general trends: (i) The unrelated examples are easy to detect, and most models show high performance for this class. (ii) The agree and the disagree examples are often misclassified as discuss by the baseline models. 


\begin{tabular}{|c|c|c|}
\hline \multicolumn{3}{|c|}{ Claim 1: man saved from bear attack - thanks to his justin bieber ringtone } \\
\hline Evidence Id & $\mathbf{P}_{\text {cnn }}^{\mathbf{j}}$ & Evidence Snippet \\
\hline $2069-3$ & 0.89 & $\begin{array}{l}\ldots \text { fishing in the yakutia republic, russia, igor vorozhbitsyn is lucky to be alive after } \\
\text { his justin bieber ringtone, baby, scared off a bear that was attacking him }{ }^{0.41} \ldots\end{array}$ \\
\hline $2069-7$ & 1.0 & $\begin{array}{l}\text {.. but as the bear clawed vorozhbitsyn ' } \mathrm{s} \text { face and back his mobile phone rang } \\
\text {, the ringtone selected was justin bieber ' } \mathrm{s} \text { hit song baby . rightly startled }{ }^{\mathbf{1 . 0 0}} \text {, } \\
\text { the bear retreated back into }{ }^{\mathbf{0 . 3 9}} \text { the forest ... }\end{array}$ \\
\hline \multicolumn{3}{|c|}{ true label: agree; predicted label: agree } \\
\hline \multicolumn{3}{|c|}{ Claim 2: 50ft crustacean, dubbed crabzilla, photographed lurking beneath the waters in whitstable } \\
\hline Evidence Id & $\mathbf{P}_{\text {cnn }}^{\mathbf{j}}$ & Evidence Snippet \\
\hline $24835-1$ & 0.0046 & ... a marine biologist has killed off claims ${ }^{-\mathbf{0 . 0 0 0 8}}$ that a giant crab is ${ }^{\mathbf{0 . 0 0 3 3}}$ living on the \\
\hline $24835-7$ & -0.0008 & $\begin{array}{l}\text { kent coast - insisting the image is probably a well - doctored hoax }{ }^{0.0012} \ldots \\
\ldots \text { i don' } t \text { know what the currents are like around that harbour or what sort of they might } \\
\text { produce in the sand, but } i \text { think it' } s \text { more conceivable that someone is playing } 0.0007 \\
\text { about with the photo ... }\end{array}$ \\
\hline
\end{tabular}

Table 3: Examples of highly ranked snippets of evidence for an input claim, which are automatically extracted by our inference component. The $P_{c n n}^{j}$ column and the values in the top-right corner of the highlighted snippets show the similarity between the claim and evidence, and between the claim and snippets of the evidence, respectively.

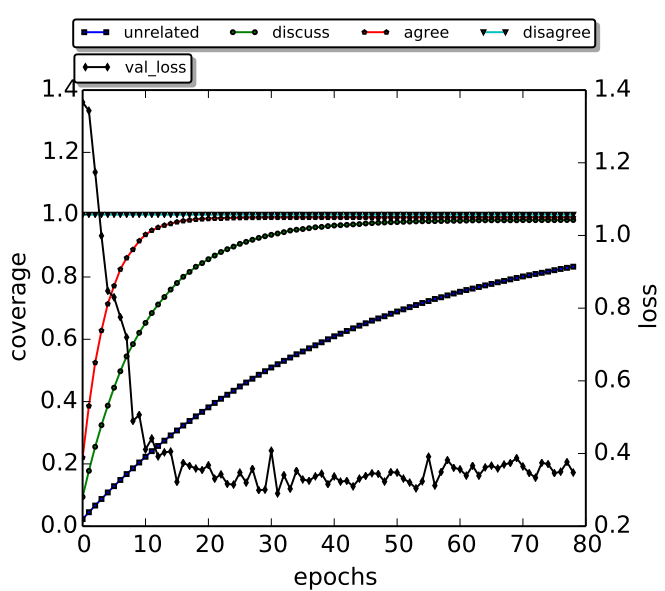

Figure 4: Effect of data coverage. The left $y$-axis shows the fraction of data observed during training (coverage), the right $y$-axis shows the loss during training.

This is mainly because the document that discusses a claim often shares the same topic with the claim, but then it does not take a stance with respect to that claim. (iii) The disagree examples are the most difficult ones for all models, probably because they represent by far the smallest class.

\section{Discussion}

\subsection{Training Data Coverage}

As discussed previously, we balance the data at each training iteration by randomly selecting $z$ instances from each of the four target classes, where $z$ is the size of the class with the minimum number of training instances. Here, we investigate the amount of training data that gets actually used.
For this purpose, at each training iteration, we report the proportion of the training instances from each class that have been used for training so far, either at the current or at any of the previous iterations. As Figure 4 shows, our random data sampling procedure eventually covers almost all training instances. Since the disagree class is the smallest, its instances remain fully covered throughout the process. Moreover, almost all other related instances, i.e., agree and discuss, are observed during training, as well as a large fraction of the dominating unrelated examples. Note that the model achieves its best (lowest) loss on the validation dataset at iteration 31, when almost all $r e$ lated training instances are observed. This happens while the corresponding fraction for the unrelated pairs is around $50 \%$, i.e., a considerable number of the unrelated instances are not required to be used for training.

\subsection{Explainability}

One of the main advantages of our memory network model, compared to the baselines and to related work in general, is that it has the capacity to explain its predictions by extracting snippets from each piece of evidence that supports its prediction. As we explained in Section 2.3, our inference component predicts the similarity between each piece of evidence $x_{j}$ and the claim $s$ at the $n$ grams level using the similarity matrix $M^{\prime}$ and the claim-evidence similarity vector $P_{c n n}^{j}$. Below, we explore our model's explainability in more detail. 


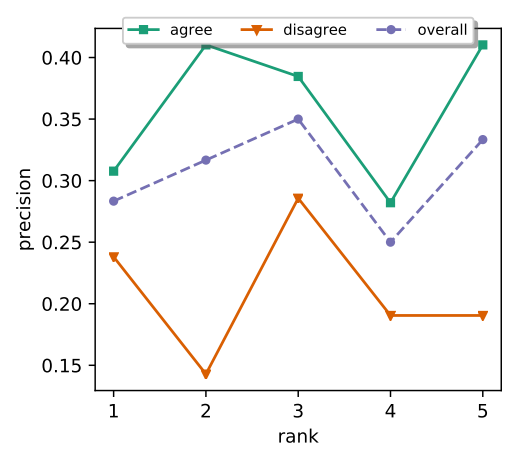

(a) $n$-grams

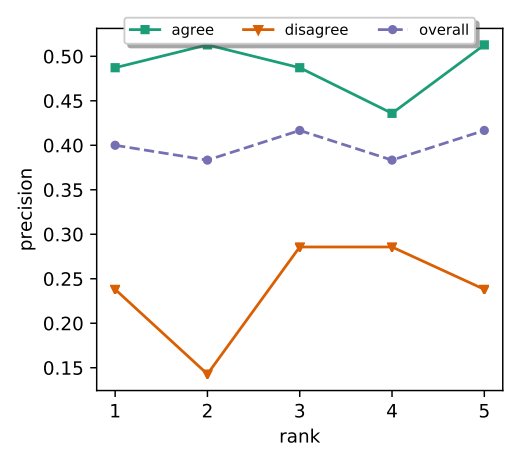

(b) consecutive $n$-grams

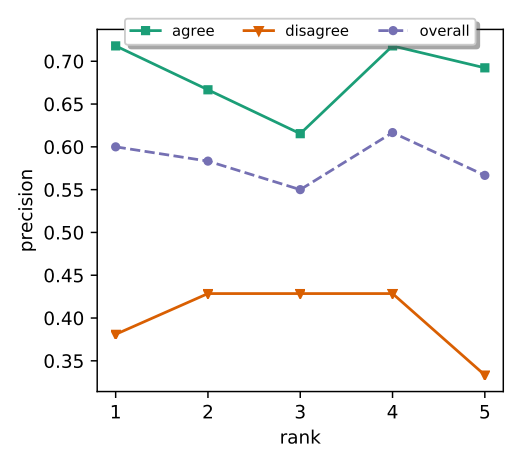

(c) sentences

Figure 5: Prediction explainability. Sub-figures (a)-(c) show the precision of our model at explaining its predictions when the pieces of evidence are (a) fixed-length $n$-grams $(n=5)$, (b) combinations of several consecutive $n$-grams with similar scores, or (c) the entire sentence, if it includes at least one extracted $n$-gram snippet.

Table 3 shows examples of two claims and the snippets extracted as evidence. Column $P_{c n n}^{j}$ shows the overall similarity between the evidence and the corresponding claim as computed by the inference component of our model. The highlighted texts are the snippets with the highest similarity to the claim as extracted by the same component. The values on the snippets' top-right show the claim-snippet similarity values obtained by the inference component.

Note that all snippets are fixed-length, namely 5-grams; however, in case there are several consecutive $n$-grams with similar scores, for better illustration, we combine them into a single snippet and we report their average values (see the snippet for evidence 2069-3). As these examples show, our model can accurately predict the stance of these pieces of evidence against their corresponding claims. Also, claim 2 and its corresponding evidence are shown at the second row of Table 3. As this example shows, the similarity values associated with snippets are either too small or negative, e.g., see the similarity value for the snippet "biologist has killed off claims." We can see that these help the model to make accurate predictions.

We conduct the following experiment to quantify the performance of our memory network at explaining its predictions: we randomly sample 100 agree/disagree claim-document examples from our gold data, and we manually evaluate the top five pieces of evidence that our model provides to support/oppose the corresponding claims. ${ }^{3}$

\footnotetext{
${ }^{3}$ In 76 cases, our model correctly classified the agree/disagree examples when the evaluation was conducted, and it further provided arguably adequate snippets.
}

Figure 5(a) shows the precision of our memory network model at explaining its predictions when each supporting/opposing piece of evidence is an $n$-gram snippet of fixed length $(n=5)$ for the agree and the disagree classes, and their combinations at the top- $k$ ranks, $k=\{1, \ldots, 5\}$. We can see in the figure that the model achieves precision of $0.28,0.32,0.35,0.25$, and 0.33 at ranks $1-5$. Moreover, we find that it can accurately identify useful key phrases such as officials declared the video, according to previous reports, believed will come, president in his tweets as supporting pieces of evidence, and proved a hoax, shot down a cnn report, would be skeptical as opposing pieces of evidence.

Note that this relatively low precision of our memory network model at explaining its agreeldisagree predictions is mainly due to the unsupervised nature of this task as no gold snippets justifying the document's gold stance with respect to the target claim are available in the Fake News Challenge dataset. ${ }^{4}$

Furthermore, our evaluation setup is at the $n$ gram level in Figure 5(a). However, if we conduct a more coarse-grained evaluation where we combine consecutive $n$-grams with similar scores into a single snippet, the precision for these new snippets will improve to $0.40,0.38,0.42,0.38$, and 0.42 at ranks $1-5$, as Figure 5(b) shows. If we further extend the evaluation to the sentence level, the precision will jump to $0.60,0.58,0.55,0.62$, and 0.57 at ranks 1-5, as we can see in Figure 5(c).

\footnotetext{
${ }^{4}$ Some other recent datasets, to be presented at this same HLT-NAACL'2018 conference, do have such gold evidence annotations (Baly et al., 2018; Thorne et al., 2018).
} 


\section{Related Work}

While stance detection is an interesting task in its own right, e.g., for media monitoring, it is also an important component for fact checking and veracity inference. ${ }^{5}$ Automatic fact checking was envisioned by Vlachos and Riedel (2014) as a multi-step process that $(i)$ identifies check-worthy statements (Hassan et al., 2015; Gencheva et al., 2017; Jaradat et al., 2018), (ii) generates questions to be asked about these statements (Karadzhov et al., 2017), (iii) retrieves relevant information to create a knowledge base (Shiralkar et al., 2017), and (iv) infers the veracity of these statements, e.g., using text analysis (Castillo et al., 2011; Rashkin et al., 2017) or information from external sources (Mihaylova et al., 2018; Karadzhov et al., 2017; Popat et al., 2017).

There have been some nuances in the way researchers have defined the stance detection task. SemEval-2016 Task 6 (Mohammad et al., 2016) targets stances with respect to some target proposition, e.g., entities, concepts or events, as infavor, against, or neither. The winning model in the task was based on transfer learning: a Recurrent Neural Network trained on a large Twitter corpus was used to predict task-relevant hashtags and to initialize a second recurrent neural network trained on the provided dataset for stance prediction (Zarrella and Marsh, 2016). Subsequently, Zubiaga et al. (2016) detected the stance of tweets toward rumors and hot topics using linear-chain conditional random fields (CRFs) and tree CRFs that analyze tweets based on their position in treelike conversational threads.

Most commonly, stance detection is defined with respect to a claim, e.g., as in the 2017 Fake News Challenge. The best system in the challenge was an ensemble of gradient-boosted decision trees with rich features (e.g., sentiment, word2vec, singular value decomposition (SVD) and TF.IDF features, etc.) and a deep convolutional neural network to address the stance detection problem (Baird et al., 2017).

Unlike the above work, we use a feature-light memory network that jointly infers the stance and highlights relevant snippets of evidence with respect to a given claim.

\footnotetext{
${ }^{5}$ Yet, stance detection and fact checking are typically supported by separate datasets. Two notable upcoming exceptions, both appearing in this HLT-NAACL'2018, are (Thorne et al., 2018) for English and (Baly et al., 2018) for Arabic.
}

\section{Conclusion}

We studied the problem of stance detection, which aims to predict whether a given document supports, challenges, or just discusses a given claim. The nature of the task requires a machine learning model to focus on the relevant paragraphs of the evidence. Moreover, in order to understand whether a paragraph supports a claim, there is a need to refer to information in other paragraphs. CNNs or LSTMs are not well-suited for this task as they cannot model complex dependencies such as semantic relationships with respect to entire previous paragraphs. In contrast, memory networks are exactly designed to remember previous information. However, given the large size of documents and paragraphs, basic memory networks do not handle well irrelevant and noisy information, which we confirmed in our experiments.

Thus, we proposed a novel extension of general memory networks based on a similarity matrix and a stance filtering component, which we apply at the inference level, and we have shown that this extension offers sizable performance gains making memory networks competitive. Moreover, our model can extract meaningful snippets from documents that can explain the stance of a given claim.

In future work, we plan to extend the inference component to select an optimal set of explanations for each prediction, and to explain the model as a whole, not only at the instance level.

\section{Acknowledgment}

This research was carried out in collaboration between the MIT Computer Science and Artificial Intelligence Laboratory (CSAIL) and the HBKU Qatar Computing Research Institute (QCRI).

\section{References}

Sean Baird, Doug Sibley, and Yuxi Pan. 2017. Talos targets disinformation with fake news challenge victory. https://blog.talosintelligence.com/2017/06/ talos-fake-news-challenge.html.

Ramy Baly, Mitra Mohtarami, James Glass, Lluís Màrquez, Alessandro Moschitti, and Preslav Nakov. 2018. Integrating stance detection and fact checking in a unified corpus. In Proceedings of HLT-NAACL. New Orleans, LA, USA.

Carlos Castillo, Marcelo Mendoza, and Barbara Poblete. 2011. Information credibility on Twitter. In Proceedings of $W W W$. Hyderabad, India, pages 675-684. 
Jeff Donahue, Lisa Anne Hendricks, Sergio Guadarrama, Marcus Rohrbach, Subhashini Venugopalan, Trevor Darrell, and Kate Saenko. 2015. Long-term recurrent convolutional networks for visual recognition and description. In Proceedings of CVPR. Boston, MA, USA, pages 2625-2634.

Pepa Gencheva, Preslav Nakov, Lluís Màrquez, Alberto Barrón-Cedeño, and Ivan Koychev. 2017. A context-aware approach for detecting worthchecking claims in political debates. In Proceedings of RANLP. Varna, Bulgaria, pages 267-276.

Ian J. Goodfellow, David Warde-Farley, Mehdi Mirza, Aaron Courville, and Yoshua Bengio. 2013. Maxout networks. In Proceedings of ICML. Atlanta, GA, USA, pages 1319-1327.

Naeemul Hassan, Chengkai Li, and Mark Tremayne. 2015. Detecting check-worthy factual claims in presidential debates. In Proceedings CIKM. Melbourne, Australia, pages 1835-1838.

Israa Jaradat, Pepa Gencheva, Alberto Barrón-Cedeño, Lluís Màrquez, and Preslav Nakov. 2018. ClaimRank: Detecting check-worthy claims in Arabic and English. In Proceedings of HLT-NAACL. New Orleans, LA, USA.

Georgi Karadzhov, Preslav Nakov, Lluís Màrquez, Alberto Barrón-Cedeño, and Ivan Koychev. 2017. Fully automated fact checking using external sources. In Proceedings of RANLP. Varna, Bulgaria, pages 344-353.

Todor Mihaylov, Georgi Georgiev, and Preslav Nakov. 2015. Finding opinion manipulation trolls in news community forums. In Proceedings of CoNLL. Beijing, China, pages 310-314.

Todor Mihaylov and Preslav Nakov. 2016. Hunting for troll comments in news community forums. In Proceedings of ACL. Berlin, Germany.

Tsvetomila Mihaylova, Preslav Nakov, Lluis Marquez, Alberto Barron-Cedeno, Mitra Mohtarami, Georgi Karadzhov, and James Glass. 2018. Fact checking in community forums. In Proceedings of AAAI. New Orleans, LA, USA.

Saif Mohammad, Svetlana Kiritchenko, Parinaz Sobhani, Xiao-Dan Zhu, and Colin Cherry. 2016. SemEval-2016 task 6: Detecting stance in tweets. In Proceedings of SemEval. Berlin, Germany, pages 31-41.

Jeffrey Pennington, Richard Socher, and Christopher Manning. 2014. Glove: Global Vectors for Word Representation. In Proceedings of EMNLP. Doha, Qatar, pages 1532-1543.

Kashyap Popat, Subhabrata Mukherjee, Jannik Strötgen, and Gerhard Weikum. 2017. Where the truth lies: Explaining the credibility of emerging claims on the web and social media. In Proceedings of $W W W$. Perth, Australia, pages 1003-1012.
Hannah Rashkin, Eunsol Choi, Jin Yea Jang, Svitlana Volkova, and Yejin Choi. 2017. Truth of varying shades: Analyzing language in fake news and political fact-checking. In Proceedings of EMNLP. Copenhagen, Denmark, pages 2931-2937.

Tara N Sainath, Oriol Vinyals, Andrew Senior, and Haşim Sak. 2015. Convolutional, long short-term memory, fully connected deep neural networks. In Proceedings of ICASSP. Brisbane, Australia, pages 4580-4584.

Prashant Shiralkar, Alessandro Flammini, Filippo Menczer, and Giovanni Luca Ciampaglia. 2017. Finding streams in knowledge graphs to support fact checking. In Proceedings of ICDM. New Orleans, LA, USA, pages 859-864.

Karen Spärck Jones. 2004. IDF term weighting and IR research lessons. Journal of documentation 60(5):521-523.

Sainbayar Sukhbaatar, Arthur Szlam, Jason Weston, and Rob Fergus. 2015. End-to-end memory networks. In Proceedings of NIPS. Montreal, Canada, pages 2440-2448.

Ming Tan, Cicero dos Santos, Bing Xiang, and Bowen Zhou. 2016. Improved representation learning for question answer matching. In Proceedings of ACL. Berlin, Germany, pages 464-473.

James Thorne, Andreas Vlachos, Christos Christodoulopoulos, and Arpit Mittal. 2018. FEVER: A large-scale dataset for fact extraction and VERification. In Proceedings of HLT-NAACL. New Orleans, LA, USA.

Andreas Vlachos and Sebastian Riedel. 2014. Fact checking: Task definition and dataset construction. In Proceedings of the ACL 2014 Workshop on Language Technologies and Computational Social Science. Baltimore, MD, USA, pages 18-22.

Soroush Vosoughi, Deb Roy, and Sinan Aral. 2018. The spread of true and false news online. Science 359(6380):1146-1151.

Guido Zarrella and Amy Marsh. 2016. MITRE at SemEval-2016 Task 6: Transfer learning for stance detection. In Proceedings of SemEval. San Diego, CA, USA, pages 458-463.

Arkaitz Zubiaga, Elena Kochkina, Maria Liakata, Rob Procter, and Michal Lukasik. 2016. Stance classification in rumours as a sequential task exploiting the tree structure of social media conversations. In Proceedings of COLING. Osaka, Japan, pages 24382448.

Zhen Zuo, Bing Shuai, Gang Wang, Xiao Liu, Xingxing Wang, Bing Wang, and Yushi Chen. 2015. Convolutional recurrent neural networks: Learning spatial dependencies for image representation. In Proceedings of CVPR. Boston, MA, USA, pages 18-26. 